\title{
Intramucosal Adenocarcinoma
}

National Cancer Institute

\section{Source}

National Cancer Institute. Intramucosal Adenocarcinoma. NCI Thesaurus. Code C95397.

A neoplastic lesion that shows morphologic evidence of invasion into the lamina propria or muscularis mucosa. There is no evidence of invasion into the submucosa. Evidence of invasion may refer to stromal invasion by sing le cells or clusters of cells, presence of atypical and complex glandular architectural patterns beyond those that are present in normal mucosa, desmoplasia, and/or vascular invasion. 